\title{
LASER IMAGING APPROACH FOR PORTABLE BTF MEASUREMENT SYSTEM AUTOMATIC ALIGNMENT
}

\author{
Jan Hošek ${ }^{1}$, Vlastimil Havran², Jiř́ Čáp ${ }^{3}$, Šárka Němcová ${ }^{4}$ \\ 1,3,4 Czech Technical University \\ Technická 4, 16607 Praha 6, Czech Republic \\ ${ }^{2}$ Czech Technical University \\ Karlovo nám. 13, 12135 Praha 2, Czech Republic \\ Jan.Hosek@fs.cvut.cz, havran@fel.cvut.cz,_iri.Cap@fs.cvut.cz, Sarka.Nemcova@fs.cvut.cz
}

\begin{abstract}
The paper presents a new alignment sensing possibility for automation of BTF instrument with regards to the sample surface. We have developed a portable bidirectional texture function (BTF) measurement instrument allowing for on-site measurement outside the laboratory. It is a newly emerging technique enabling data acquisition of the real objects without the necessity of their extraction from the environment. A practical issue of the sample measurement is the instrument alignment against the measured surface. The system is provided with five degrees of freedom to be able to set the right position of the instrument to the sample surface without the necessity of the sample manipulation. We used a laser auto-collimation principle to provide feedback information for the system alignment. Now we propose a new approach for acquisition of the feedback information for the system alignment based on laser-imaging approach. We present theoretic relations of the proposed system and technical demands to reach the desired uncertainty of the system misalignment to the sample surface of less than $0.1^{\circ}$.
\end{abstract}

Keywords: System Alignment, Autocollimator, Laser Adjustment, BTF Measurement.

\section{Introduction}

The measurement system alignment towards the measured sample is the crucial task for quantitative measurement instruments, while geometrical misalignments affect the level of the measured data uncertainty generally. A convergent approach of the system alignment requires feedback information used as an input for the next steps of the system adjustment process. We have developed a portable bidirectional texture function (BTF) measurement instrument allowing on-site measurement outside the laboratory. The system was designed to acquire large value of high-dynamic-range images of the sample surface from different points of view and points of illumination in the shortest possible time. The system was designed as a portable system which can take measurement of the real object samples without the necessity of their extraction from the environment [1,2]. It requires an exact alignment of the system with regard to the sample to acquire reliable BTF data of the sample surface. We provided the system with five degrees of freedom to be able to set the right position of the instrument to the sample surface without the necessity of the sample manipulation. As feedback information for system alignment we used a laser auto-collimation. The system reaches a high level of angular resolution to allow for a correct system alignment with required uncertainty. We have found some drawbacks of this auto-collimating principle, which deteriorate the instrument alignment automation. The first drawback is that the laser auto-collimator provides feedback information just about the angular orientation of the instrument to the sample surface.

The axial position of the system to the sample is acquired through the imaging system and marker stickers and image registration method [2]. The main drawback of the autocollimation method is the necessity to use the mirror which represents the sample surface as the majority of the samples scatter the light. We aim to eliminate a mirror used for retro-reflection due to following reasons:

a) The instrument angular position is affected by the misalignment of the mirror to the sample.

b) The physical contact of the mirror to the sample can cause sample changes or destruction.

c) It is tricky to place and remove the mirror from the sample in a small gap $(1 \mathrm{~mm})$ between the sample and instrument of the $600 \mathrm{~mm}$ diameter.

d) Mirror removal deteriorates the possibility of the system alignment automation.

Due to those reasons we analysed other possibilities to achieve feedback information for the system alignment. We propose a new approach for the acquisition of the feedback information for the system alignment based on laser imaging approach, where lasers incident directly to the scattering 
surface sample and no mirror is needed. We present theoretic relations of the proposed system and technical demands to reach desired uncertainty of the system misalignment to the sample surface less than $0.1^{\circ}$.

\section{The BTF Measurement Instrument}

The appearance of real surfaces depends on the kind and direction of illumination and the direction of observation, therefore the actual surface perception depends on the conditions of its observation. This is a problem in virtual representation of the surface appearance in digital visualization. There exist few concepts of digital representation of the real surfaces. The surface reflectance of a single point was formalized by Nicodemus et al. [3] as bidirectional reflectance distribution function, (BRDF). This is a four-dimensional function, where two values specify the direction of incident light and two the direction of outgoing light. A faithful representation of the $2 \mathrm{D}$ surface needs to be represented by a set of thousands of images taken under various combinations of illumination and observation directions. Such a spatially varying description of surface reflectance, a sevendimensional function, is called bidirectional texture function (BTF) and was introduced by Dana et al. [4].

It is technically difficult to realize an instrument capable of acquiring thousands of images with different illumination and observation directions of the sample surface within reasonable time, so there is only few realized BTF setups. Portable BTF measurement setups are rare, but a few can be found that could be realized as portable ones $[5,6,7,8,9,10,11]$. Our instrument for BTF on site measurement was designed as a compact and portable system which tackles the dimension issues and acquisition speed enhancement by a combination of the parallelization of the illuminators and cameras with their mutual motions regarding the sample surface [1]. The system is equipped with totally 139 LEDs illuminating the sample surface during a measurement sequence. Surface appearance data are acquired with 6 cameras simultaneously. Due to practical reasons the single sample measured data are limited to 16,680 highdynamic-range images taken at 20 different rotational positions taken within 1020 s. The system was designed to measure at maximum elevation angle $75^{\circ}$ which requires for a measured sample size the depth of field $51 \mathrm{~mm}$. For reliable data acquisition we evaluated the uncertainty of the sample illumination beam angle as $\pm 0.59^{\circ}$, mainly caused by high fabrication tolerance of the LED chip inclination. The maximum camera total angular uncertainty was calculated to be $\pm 0.167^{\circ}$ in the meridional direction and $\pm 0.184^{\circ}$ in the zonal direction. These uncertainties are valid under the assumption of maximum misalignment of the measurement instrument regarding the sample within the range $\pm 0.5 \mathrm{~mm}$ and the rotary axis perpendicular towards the sample inclination $\pm 0.1^{\circ}$.

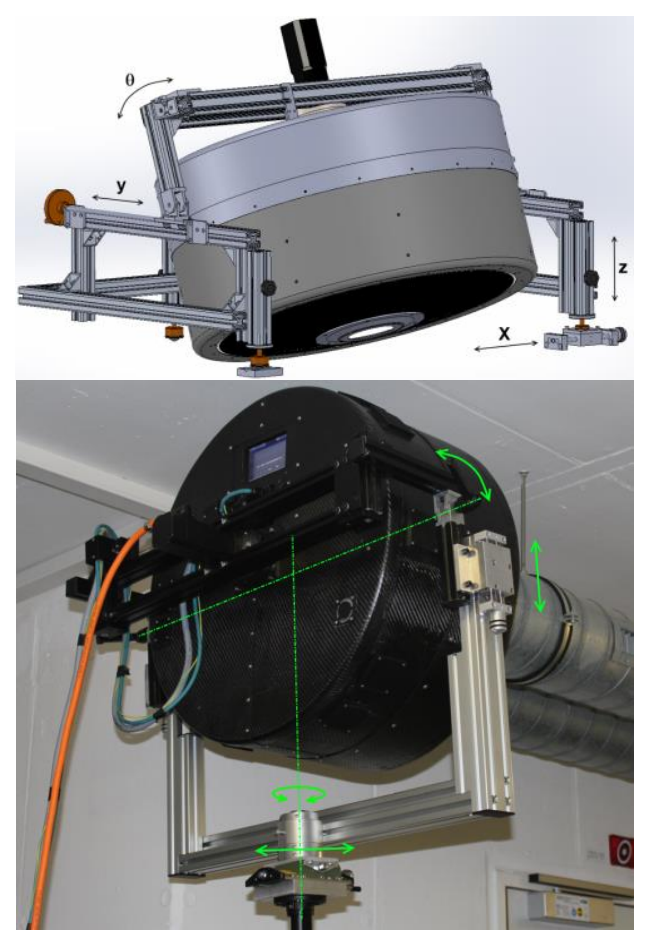

Figure 1: The 3D model of the "table-top" adjusting system - top, and realization of the U-shaped external frame adjusting system - bottom.

These conditions raise demands on the possibility to properly adjust the instrument with regards to the measured surface. Due to this demands we provided the BTF measurement instrument with five degrees of freedom to be able to set the right position of the instrument to the sample surface as the sample is fixed in a space and cannot move. The system can be mounted into one of two external frames allowing the system to the sample adjustment. The first option is an U-shaped frame used for a sample orientation from the wall $\left(90^{\circ}\right)$ up to the ceiling $\left(180^{\circ}\right)$. The second option is the "tabletop" frame used for sample orientation from the ground $\left(0^{\circ}\right)$ up to the wall $\left(90^{\circ}\right)$. The $3 \mathrm{D}$ model and realized system are shown in the Figure 1.

The desired level of instrument-sample alignment was achieved with feedback information provided by our design of laser autocollimator schematically shown in the Figure 2.

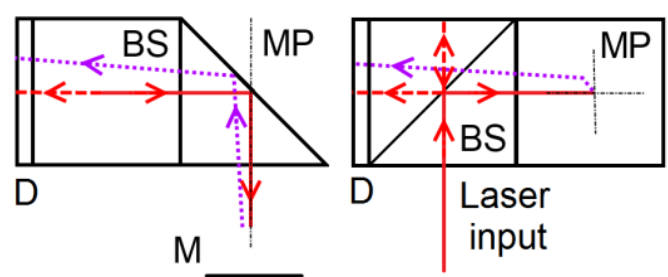

Figure 2: The laser autocollimator principle, the sideview - left, the top view - right. 
The laser autocollimator consists of an adjustable 1 $\mathrm{mW}$ laser module and a lateral displacement beamsplitter made of a beamsplitter cube (BS) and $90^{\circ}$ mirror prism (MP). The laser beam is split into a reference beam making a fixed laser spot on a diffuser attached to the beamsplitter prism and a measurement beam aligned with the instrument rotary axis. The sample surface temporarily provided with a thin mirror (M) reflects the beam back through the prisms to the diffuser (D). The angular misalignment is indicated as a distance between the reflected and the reference laser spots imaged with 5 Mpixel camera. On $25 \mathrm{~mm}$ wide diffuser, with the light travel distance $346 \mathrm{~mm}$ between the mirror and the diffuser, we can detect angle changes in the range $\pm 2.65^{\circ}$ with a theoretical resolution of $0.0027^{\circ}$. The feedback information provided by the autocollimator helps to set the instrument rotary axis perpendicularly towards the sample with an accuracy of $\pm 0.1^{\circ}$.

\section{A new Alignment Sensing Approach}

We were looking for another suitable gauge for the instrument-sample alignment to get rid of the mirror which deteriorates the possibility of the system adjustment automation. We analysed possibilities of a laser imaging system where sampleinstrument position and angular misalignment can be assessed with imaging of the laser spot incident to the scattering sample surface. We treat the alignment as two independent coordinate systems that can move towards each other in five degrees of freedom.

The basic system is connected with the instrument rotational axis and fixed object distance. The object distance cross a laser beam in meridional plane inclined by the angle $\omega$ to the axis at the radial distance $a$. The laser beam can turn around the axis as the instrument is turning above the sample. The second coordinate system represents the sample surface where the laser beam is incident in.

This coordinate system can be translated with regard to the first coordinate system in three axes by distances $\left(x, y_{r}, z_{r}\right)$ and tilt by angles $\varphi$ and $\theta$ in XZ and $X Y$ planes, respectively. The laser spot on the sample surface is viewed by a distal camera placed in the instrument rotational axis thus just XY plane spot position can be evaluated.

When the instrument rotates around the axis perpendicular to the sample surface, the laser spot circumscribes a circle of the radius $a$. If any linear or angular misalignment between the two coordinate systems is present the circumscribed curve becomes an ellipsis.

An example is shown in the Figure 3.
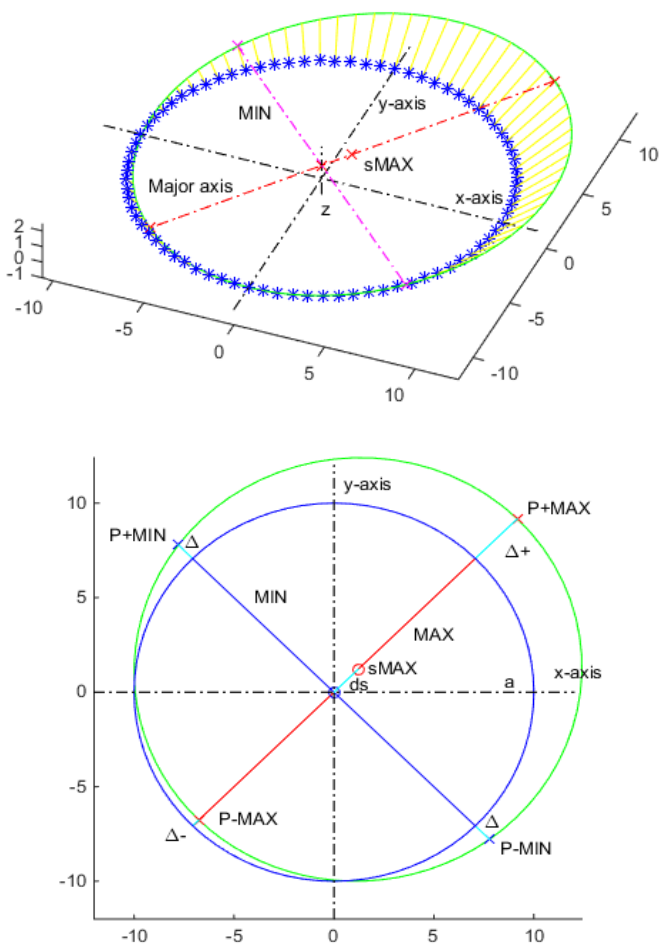

Figure 3: 3D view to trajectory of the laser beam in the object plane (blue) and its projection to tilted sample plane (green) - top, 2D to view projection of the laser spot trajectory to the object plane - bottom.

By analysis of the ellipsis shape, the individual misalignments of the coordinate systems can be evaluated. The ellipsis can be sampled by any $N$ angular position of the instrument rotation, but it is advantageous to acquire laser spot data for four angular positions $0,90,180$ and 270 degrees with respect to each angular sample $N_{i}$. This simplifies the evaluation of the major axis of the ellipsis angle $\varepsilon_{\text {MAX }}$ as an angle of the maximum distance $M A X$ of the spot position in under angular turn by $\Delta \varepsilon^{\prime}=180^{\circ}$.

Perpendicular to the major axis one can find a minimum distance $M I N$ between two spots turned by $180^{\circ}$. The centres of $M A X$ and $M I N$ distances make a vector crossing the rotational axis of the instrument.

The centres are evaluated by relations:

$$
\begin{gathered}
s_{M A X}=\frac{P_{M A X}^{+}+P_{M A X}^{-}}{2} \\
s_{M I N}=\frac{P_{M I N}^{+}+P_{M I N}^{-}}{2}
\end{gathered}
$$

If the misalignments of the two coordinate systems are small the centre of the minimum distance $S_{M I N}$ is on the instrument rotational axis and $M I N$ dimension is not affected by angular tilts. The $M I N$ value is strongly affected by misalignment of the systems in $Z$ axis and negligibly affected by radial distance misalignment. 
A cross-section in the plane given by the major axis of the ellipsis and the instrument rotational axis is depicted in the Figure 4.

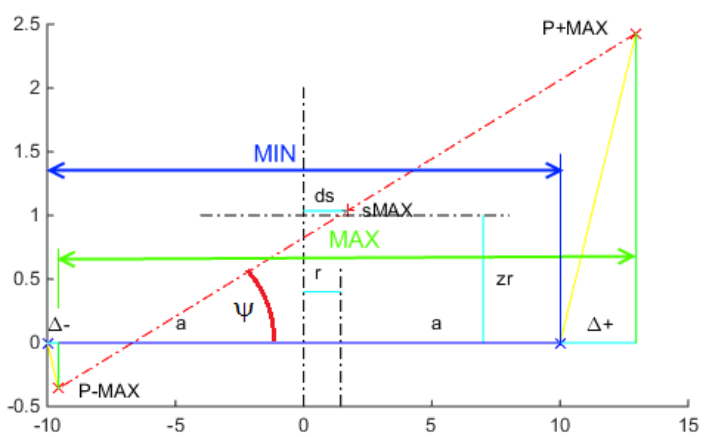

Figure 4: Sample (red) and object (blue) planes in the laser trajectory ellipsis major plane.

If we turn the MIN distance plane into the major plane of the ellipsis around the instrument rotational axis, we can evaluate distances $\Delta^{-}$and $\Delta^{+}$between $M A X$ and $M I N$ distances endpoints and distance $d s$ between its centres. While the coordinate system of the sample plane is radially moved out of the rotational axis by $r$, where:

$$
r=\frac{x_{r}}{\cos \varepsilon}=\frac{y_{r}}{\sin \varepsilon}
$$

We can evaluate this radial shift from relations:

$$
\begin{gathered}
\tan \psi=\frac{h^{-}}{\frac{M I N}{2}+r}=\frac{h^{-}}{\Delta^{-}+\frac{M A X}{2} d s+r} \\
r=\frac{M I N}{4}-\frac{M A X}{4}-\frac{\Delta^{-}}{2}+\frac{d s}{2}
\end{gathered}
$$

where $\psi$ is the tilt angle of the sample plane in the ellipsis major axis. A minor axis ellipsis distance $M R$ in the centre of the sample plane coordinate system can be achieved numerically by suitable approximation of the ellipsis or measured directly as distance of the points turned by the angles:

$$
\varepsilon_{+,-}= \pm 90^{\circ} \mp \tan ^{-1} \frac{2 r}{M I N}
$$

with regard to the major axis plane. This angle $\varepsilon$ can be used for evaluation of $\mathrm{Z}$ axis translation distance $Z_{r}$ between both coordinate systems by equation:

$$
z_{r}=\frac{M R-2 a \cos \varepsilon}{2 \cos \varepsilon \tan \omega}
$$

The remaining coordinate systems displacements $x_{r}$ and $y_{r}$ can be simply evaluated from equation (3). In the case of a small difference between $M I N$ and $M A X$ ellipsis distances the ellipsis converge to a circle and $M R$ distance can be evaluated as:

$$
M R=\frac{M I N}{\cos \varepsilon}
$$

The last coordinate systems misalignment the maximum tilt of the sample plane in the ellipsis major axis $\psi$ can be evaluated by equation:

$$
\tan \psi=\frac{M A X-M R-2 d s}{\tan \omega(2 M R-M A X+2 d s)}
$$

This approach gives values for the corrections of the both coordinate systems misalignment, without the necessity to place any mirror on the sample surface and it can be employed as inputs for automatic alignment system actuation.

\section{Implementation Conditions for the BTF Measurement System}

We need to analyse the technical conditions and resolution possibilities of the proposed approach for future implementation into our BTF measurement system. The measured sample surfaces appear different kinds of regular or random patterns, but usually with no axial symmetry. For this reason the translations between coordinate systems $x_{r}$ and $y_{r}$ are not important and can be neglected. It simplifies the problem to a more symmetric case, when minor axis of the ellipsis $M R$ depends just on $\mathrm{Z}$ axis translation $Z_{r}$ and major axis of the ellipsis $M A X$ depends on both $Z_{r}$ translation and plane inclination angle $\psi$. Cross-sections in the both major and minor planes of the ellipsis are shown in the Figure 5 and 6.

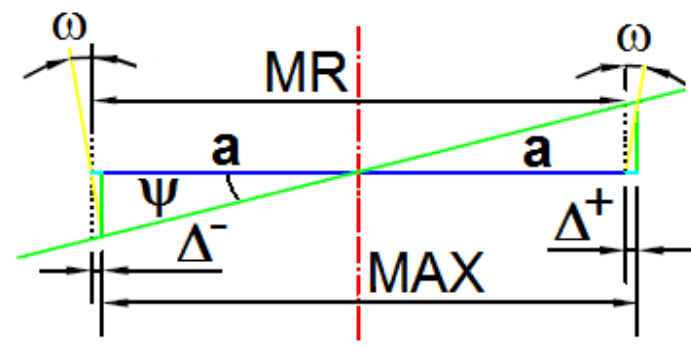

Figure 5: Sample and object planes in the laser trajectory ellipsis major plane.

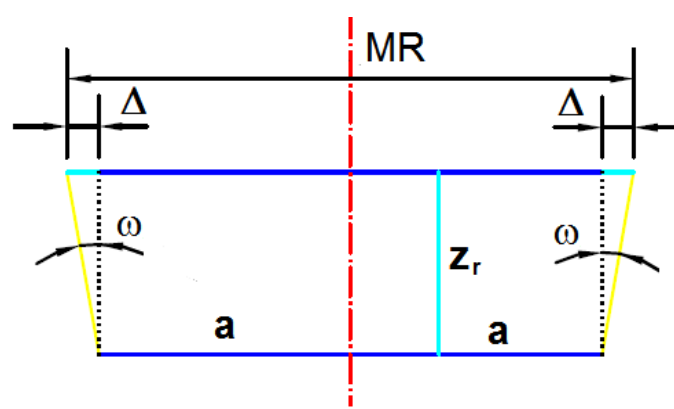

Figure 6: Sample and object planes in the laser trajectory ellipsis minor plane. 
Evaluation of both variables depends on the measurement of distances $\Delta, \Delta^{-}$and $\Delta^{+}$between important points of the ellipsis created by laser spot in to the sample plane.

We aim to find the conditions of the highest sensitivity of those distances on the individual variables. In the minor plane we can evaluate dependence:

$$
\Delta=z \tan \omega
$$

and the sensitivity $c_{z}$ as:

$$
c_{z}=\frac{\partial \Delta}{\partial z}=\tan \omega
$$

It shows that the highest sensitivity can be reached for $\omega \rightarrow 90^{\circ}$. If we compensate the $Z_{r}$ translation we can evaluate the dependence on inclination angle $\psi$ in the ellipsis major axis:

$$
\begin{aligned}
& \tan \psi=\frac{\Delta^{-}}{\tan \omega\left(a-\Delta^{-}\right)} \\
& \Delta^{-}=\frac{a}{1+\frac{1}{\tan \omega \tan \psi}} .
\end{aligned}
$$

The sensitivity $c_{\psi}$ is:

$$
c_{\psi}=\frac{\partial \Delta^{-}}{\partial \psi}=\frac{a \tan \omega\left(\tan ^{2} \psi+1\right)}{(\tan \omega \tan \psi+1)^{2}}
$$

If we take into account that we are interested in small values of angle $\psi$ the limit of the sensitivity function for $\psi \rightarrow 0^{\circ}$ is:

$$
\lim _{\psi \rightarrow 0} c_{\psi}=a \tan \omega
$$

It shows the highest sensitivity can be achieved for $\omega \rightarrow 90^{\circ}$ and $a \rightarrow \infty$.

In the case of our BTF measurement system both parameters are limited with physical dimensions of the system. The value of $a_{M A X}$ is limited by sample field of view and the angle $\omega$ is limited by ambient light shield to $\omega_{\text {MAX }}=75^{\circ}$.

The minimum distinguishable distance $\Delta_{i}$ is then given by the resolving power of the camera system used for the laser spot position acquisition.

Imaging of the $\Delta_{i}$ distance through an idealized lens is shown in the Figure 7.

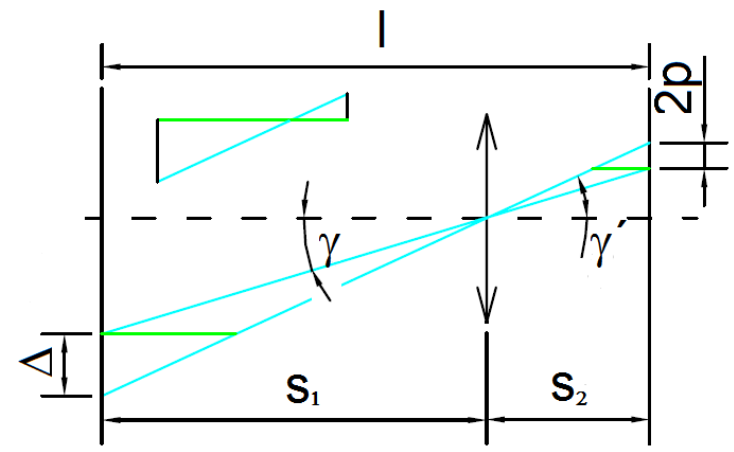

Figure 7: Imaging of distance $\Delta$ on the camera sensor with pixel pitch distance $p$ through an ideal lens.

Imaging of the object is given by imaging equation:

$$
\frac{1}{s_{1}}+\frac{1}{s_{2}}=\frac{1}{f^{\prime}}
$$

where $s_{1}$ and $s_{2}$ are distances of the object and its image, respectively, from the ideal lens of focal distance $f^{\prime}$. If the total distance between the object and its image $l=s_{1}+s_{2}$ is known, we can express this relation as:

$$
f^{\prime}=\frac{s_{1}}{l}\left(l-s_{1}\right)
$$

Keeping in mind the Nyquist-Shannon sampling theorem, that frequency needs to be sampled with at least doubled sampling frequency, a spatial frequency corresponding to the distance under resolution $\Delta$ needs to be sampled with a distance corresponding to a double of the pixel pitch distance $p$. Then we can get a relation:

$$
\frac{2 p}{\Delta}=\frac{s_{2}}{s_{1}}=\frac{l}{s_{1}}-1 .
$$

The lens' focal distance $f^{\prime}$ needed for resolution of the desired distance $\Delta$ in the object plane at distance $l$ by a camera with a pixel pitch $p$ can be evaluated:

$$
f^{\prime}=\left(l-\frac{l \Delta}{2 p+\Delta}\right) \frac{\Delta}{2 p+\Delta} .
$$

It supposes that the lens highest transfer frequency corresponds to the pixel pitch distance space frequency $1 / p$. Due to diffraction limit the resolution of desired $\Delta$ distance put demands on F-number of the lens $f \#$ which needs to fulfil the condition:

$$
f \#<\frac{f^{\prime} \Delta}{1.22 \lambda}
$$

where $\lambda$ is the longest wavelength used for imaging. 


\section{Conclusions}

We proposed a new approach for the evaluation of misalignment between the rotational axis of the BTF measurement instrument and the sample surface generally shifted and inclined with regards to the instrument coordinate system. The system is based on a camera sensing of the position of a laser spot on incident to the sample surface during rotation of the system around its working axis. We derived conditions of the laser beam direction to achieve the highest sensitivity to the coordinate systems misalignments.

The laser beam needs to cross the instrument rotational axis under as large as possible angle $\omega$ and at as large as possible distance from the axis $a$ in the sample plane. We also evaluated necessary values of the imaging lens focal distance $f^{\prime}$ needed for resolution of desired distance $\Delta$ and the maximum Fnumber of the lens to fulfil diffraction limit condition.

\section{Acknowledgements}

This work was supported by the Czech Science Foundation of the Czech Republic, under research project number GA14-19213S and SGS17/176/OHK2/3T/12.

\section{References}

[1] J. Hosek, V. Havran, S. Nemcova, J. Bittner, and J. Cap. 2017. Optomechanical design of a portable compact bidirectional texture function measurement instrument. Appl. Opt. 56, 4 (2017), 1183-1193.

[2] V. Havran, J. Hošek, S. Němcová, J. Čáp, and J. Bittner. 2017. Lightdrum - Portable Light Stage for Accurate BTF Measurement on Site. Sensors 17, 3 (2017).
[3] F.E. Nicodemus, J.C. Richmond, J. J. Hsia, I.W. Ginsberg and T. Limperis, Geometric Considerations and Nomenclature for Reflectance; Monograph 161, NBS (US); Jones and Bartlett Publishers: Burlington, MA, USA, 1977.

[4] K. Dana, B. Van-Ginneken, S. Nayar, J. Koenderink, Reflectance and Texture of Real World Surfaces. ACM Trans. Graph. 1999, 18, 134.

[5] J. Y. Han and K. Perlin. 2003. Measuring Bidirectional Texture Reflectance with a Kaleidoscope. ACM Trans. Graph. 22, 3 (2003), 741-748.

[6] G. Müller, J. Meseth, M. Sattler, R. Sarlette, and R. Klein. Acquisition, Synthesis, and Rendering of Bidirectional Texture Functions. Computer Graphics Forum 24, 1 (2005), 83-109.

[7] M. Holroyd, J. Lawrence, and T. Zickler. 2010. A Coaxial Optical Scanner for Synchronous Acquisition of 3D Geometry and Surface Reflectance. ACM Trans. Graph. 29, 4, Article 99 (2010).

[8] Y. Mukaigawa, S. Tagawa, J. Kim, R. Raskar, Y. Matsushita, and Y. Yagi. 2011. Hemi-spherical Confocal Imaging Using Turtleback Reflector. Springer Berlin Heidelberg, Berlin, Heidelberg, 336-349.

[9] C. Schwartz, R. Sarlette, M. Weinmann, and R. Klein. 2013. DOME II: A Parallelized BTF Acquisition System. In Proceedings of the Eurographics 2013 Workshop on Material Appearance Modelling: Issues and Acquisition (MAM '13). Eurographics Association, Aire-laVille, Switzerland, Switzerland, 25-31.

[10] J. Filip, R. Vávra, and M. Krupička. 2014. Rapid Material Appearance Acquisition Using Consumer Hardware. Sensors 14, 10 (2014), 19785-19805.

[11] J. Čáp, J. Hošek, V. Havran, Š. Němcová, and K. Macúchová, "Optomechanical design of rotary kaleidoscope for bidirectional texture function acquisition", Applied Optics Vol. 56, Issue 26, 7373-7384 (2017). 